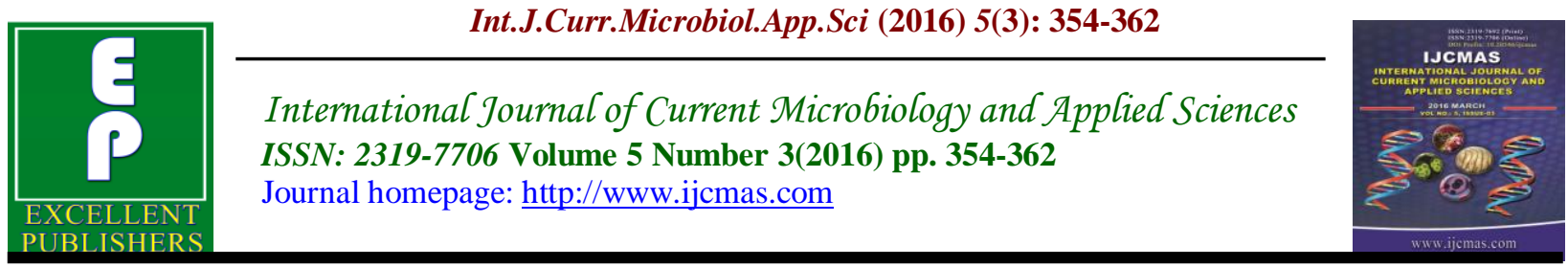

Original Research Article

http://dx.doi.org/10.20546/ijcmas.2016.503.042

\title{
Simultaneous Determination of 17 Amino Acids in Microbial Pigments from Monascus spp By UHPLC Amino Acid Analyser Using Pre-Column Derivatization
}

\author{
R. Vidyalakshmi*, R.Paranthaman and K. Sureshkumar \\ Department of Food Safety \& Quality Testing, (ISO/IEC 17025: 2005, NABL Accredited Lab), \\ Indian Institute of Crop Processing Technology, Ministry of Food Processing Industries, \\ Govt. of India, Thanjavur-613 005 (TN), India \\ *Corresponding author
}

\begin{abstract}
A B S T R A C T
Keywords

Monascus spp,

Amino acids,

UHPLC,

Pre-column

derivatization.

Article Info

Accepted:

15 February 2016

Available Online:

10, March 2016

Microbial pigments were produced from Monascus purpureus (MTCC 410), Monascus purpureus (MTCC 369), Monascus ruber (MTCC 1880) and Monascus ruber (MTCC 2326) by submerged fermentation. The essential amino acids were determined in the pigment extract natural pigments by UHPLC method using precolumn derivatization method with o-phthalaldehyde2-mercaptoethanol. The results shown in the Monascus purpureus (MTCC 410) produced the mamimum amount of amino acids, viz, Aspartic Acid ( 5.90 $\mathrm{mg} / \mathrm{L})$, Glutamic Acid (1.30 mg/L), Serine (1.80 mg/L), Histldine $(0.60 \mathrm{mg} / \mathrm{L})$, Tyrosine (5.70 mg/L), Cystine (0.80 mg/L), Valline ( $2.40 \mathrm{mg} / \mathrm{L})$, Leucine $(0.40$ $\mathrm{mg} / \mathrm{L})$ and Proline $(88.20 \mathrm{mg} / \mathrm{L})$. Analysis of these amino acids may be of great importance to determine food safety and consumer safety.
\end{abstract}

\section{Introduction}

The increasing interest in the beneficial ingredients of Monascus derived products and the attempt of decreasing the toxic components for natural colorants in food make the progress of research in the exploitation of fungal biotechnology. There are two reasons for the determination of amino acids in foods: their potential toxicity and a possibility of using them as food quality indicators. Some of the major applications of amino acids analysis are; quality control of raw materials, intermediates and end products, monitoring fermentation processes, process control and research \& development (Onal, 2007). UHPLC with precolumn derivatization is the mostly frequently reported technique for Amino acids separation and quantification (Bomke et al., 2009). This paper presents a method to determine the amino acids by UHPLC using precolum derivatization method with o-phthalaldehyde-2- 
mercaptoethanol in pigments from Monascus purpureus (MTCC 410), Monascus purpureus (MTCC 369), Monascus ruber (MTCC 1880) and Monascus ruber (MTCC 2326).

\section{Materials and Methods}

\section{Monascus Pigment Production Using Submerged Fermentation}

Yeast phosphate soluble starch broth was prepared and sterilized at $121^{\circ} \mathrm{c}$ at $15 \mathrm{lbs}$ for 15 minutes. After sterilization, the medium was inoculated with the Monascus purpureus (MTCC 410), Monascus purpureus (MTCC 369), Monascus ruber (MTCC 1880) and Monascus ruber (MTCC 2326) and incubated for 7-15 days at 25$30^{\circ} \mathrm{C}$. After incubation period the culture was sterilized at $121^{\circ} \mathrm{c}$ at $15 \mathrm{lbs}$ pressure for 15 mins. After sterilization, the broth was filtered through Whatmann No. 1 filter paper.

\section{Determination of Amino Acid in Pigments by UHPLC Amino Acid Analyser}

\section{Standard solutions}

A solution of Amino acids standards from Sigma (AAS-18) solution containing 2.5, 5.0 and $7.5 \mathrm{mg} / \mathrm{L}$ (Aspartic Acid, Glutamic Acid, Serine, Histldine, Glyeine, LThreonine, Arginine, Alanine, Tyrosine, Cystine, Valline, Methionine, Phyenylalanine, Isoleucine Leucine, Lysine, Proline) was prepared for calibration curves.

\section{Hydrolysis of samples}

The method of Zhaolai Dai et al., for amino acid extraction was followed with modification. The pigment samples were filtered through a $0.45 \mathrm{~mm}$. The samples were then hydrolyzed with $\mathrm{HCl} 6 \mathrm{M}$ at $150^{\circ} \mathrm{C}$ for $6 \mathrm{~h}$. After hydrolysis, the acid was removed by rotary evaporation. Sample was resuspended in $2 \mathrm{~mL}$ of sodium citrate buffer at $\mathrm{pH} 2.2$.

\section{UHPLC Condition}

The amino acid is determined by UHPLC method (Rouba and Ulrich, 2013 ), Instrument : Nexera UHPLC (Shimadzu) with SIL-30AC autosampler, Column : YMC-Triart C18, $1.9 \mu \mathrm{m}(75 \mathrm{mmL} . \times 3.0$ mm I.D., $1.9 \mu \mathrm{m}$, YMC Co., Ltd.), Mobile Phase : A : $20 \mathrm{mmol} / \mathrm{L}$ Phosphate Potassium Buffer (pH 6.9), B : 45/40/15 Acetonitrile/Methanol/Water, Time Program $:$ B Conc. $11 \% \rightarrow 13 \%(0.00-3.00 \mathrm{~min}), \rightarrow$ $31 \%$ (5.00 min) $\rightarrow 37 \%$ (7.5 min), $\rightarrow 70 \%$ $(10.00 \mathrm{~min}) \rightarrow 100 \%(10.50-13.50 \mathrm{~min}), \rightarrow$ $11 \%$ (14.00 min), Flow Rate : $0.8 \mathrm{~mL} / \mathrm{min}$, Column Temp. : $35^{\circ} \mathrm{C}$, Injection Volume: 1 $\mu \mathrm{L}$, Detection : RF-20Axs Ex. at $350 \mathrm{~nm}$, Em. at $450 \mathrm{~nm}, \rightarrow$ Ex. at $266 \mathrm{~nm}, \mathrm{Em}$. at $305 \mathrm{~nm}(9.0 \mathrm{~min})$, Cell Temp.: $20{ }^{\circ} \mathrm{C}$. Flow Cell: Conventional cell.

\section{Preparation of derivatization Reagents}

Mercaptopropionic Acid- 3Mercaptopropionic Acid $10 \mu \mathrm{L}$ in $0.1 \mathrm{~mol} / \mathrm{L}$ Borate Buffer (pH 9.2) $10 \mathrm{~mL}, 2$. $o$ Phthalaldehyde SolutionL: $o$-Phthalaldehyde $10 \mathrm{mg}$ in $0.1 \mathrm{~mol} / \mathrm{L}$ Borate Buffer (pH 9.2) 5 mL.3. Fluorenyl Methyl Chloro Formate (FMOC) - Acetonitrile Solution 9-Fluorenyl Methyl Chloro Formate $4 \mathrm{mg}$ in Acetonitrile $20 \mathrm{Ml}$.

\section{Derivatization Procedure}

Mercaptopropionic Acid ,OPA and sample was taken in $(\mu \mathrm{L})$ ratio of $45: 22: 7.5$ and thoroughly mixed and kept for $1 \mathrm{~min}$. then added $10 \mu \mathrm{L}$ of FMOC and sample was injected to the UHPLC using Autosampler. 


\section{Results and Discussion}

\section{Linearity}

The linearity was established using Three concentrations of amino acid standards (Table1-2 \& Figure 1-2). The data of peak area $v s$. amino acid concentration were treated by linear least squares regression analysis. The linearity data obtained should obey the equation $y=b x+a$, where $a$ is zero within the $95 \%$ confidence limits, and the coefficient of determination $\left(R^{2}\right)$ is greater than 0.984 (Armagan onal et al., 2013 \& Perucho et.al., 2015). The retention time of amino acid standards show in UHPLC, are L-Aspartic acid (Rt -0.453), L-Glutamic acid (Rt -0.667), L-Serine (Rt - 0.97) LHistidine (Rt-1.418), Glycine (Rt -1.915), Threonine (Rt- 2.372), Arginine (Rt-2.772), Alanine (Rt-3.16) ,Tyrosine 3.693, Cystine 5.185, Valine (Rt-6.269), Methionine (Rt7.847) ,Phenylalanine (Rt-8.579), Isoleucine (Rt-8.986), Leucine (Rt-9.848), Lysine (Rt-
9.988) and Proline (Rt-11.198) (Table 3-6).

The results shown in the Monascus purpureus (MTCC 410) produced the maximum amount of amino acids, Aspartic Acid (5.90 mg/L), Glutamic Acid (1.30 $\mathrm{mg} / \mathrm{L})$, Serine $(1.80 \mathrm{mg} / \mathrm{L})$, Histidine $(0.60$ $\mathrm{mg} / \mathrm{L})$, Tyrosine $(5.70 \mathrm{mg} / \mathrm{L})$, Cystine $(0.80$ $\mathrm{mg} / \mathrm{L}$ ), Valline ( $2.40 \mathrm{mg} / \mathrm{L})$, Leucine ( 0.40 $\mathrm{mg} / \mathrm{L})$ and Proline $(88.20 \mathrm{mg} / \mathrm{L})$ followed by the Monascus ruber (MTCC 2326) and given in table 1.The analytical column they used was a $3 \mu \mathrm{m}$ C18 column and the chromatogram run time is only $11 \mathrm{~min}$. Although total run time was $17 \mathrm{~min}$, this paper which describes a reliable HPLC method for the quantitation of plasma amino acids in such a short time. Although the simple sample preparation with OPA as derivatization reagent and the short analysis time, make this method very appropriate for fast usual analysis of microbial samples (Figure.1-5).

Table.1 Summary of Amino Acid Content in Monascus Pigments

\begin{tabular}{|c|c|c|c|c|}
\hline \multirow[b]{2}{*}{ Amino acids } & \multicolumn{4}{|c|}{ Amino acids Conc. (mg/L) } \\
\hline & $\begin{array}{l}\text { Monascus purpureus } \\
\text { (MTCC 410) }\end{array}$ & $\begin{array}{c}\text { Monascus } \\
\text { purpureus (MTCC } \\
\text { 369) }\end{array}$ & $\begin{array}{l}\text { Monascus ruber } \\
\text { (MTCC 1880) }\end{array}$ & $\begin{array}{l}\text { Monascus ruber } \\
\text { (MTCC 2326) }\end{array}$ \\
\hline Aspartic Acid & 5.90 & 0.00 & 6.50 & BDL \\
\hline Glutamic Acid & 1.30 & 1.40 & 2.20 & 1.90 \\
\hline Serine & 1.80 & 1.40 & 1.40 & 1.70 \\
\hline Histldine & 0.60 & 0.60 & 0.90 & 1.00 \\
\hline Glyeine & BDL* & 2.40 & 2.80 & 2.50 \\
\hline L-Threonine & BDL* & BDL* & BDL* & BDL* \\
\hline Arginine & BDL* & 0.30 & BDL* & BDL* \\
\hline Alanine & BDL* $^{*}$ & $\mathrm{BDL}^{*}$ & $\mathrm{BDL}^{*}$ & $\mathrm{BDL}^{*}$ \\
\hline Tyrosine & 5.70 & 5.00 & 3.40 & 4.00 \\
\hline Cystine & 0.80 & 0.30 & 0.20 & 0.30 \\
\hline Valline & 2.40 & 2.00 & 2.50 & 4.30 \\
\hline Methionine & BDL* & 0.00 & 9.70 & 20.80 \\
\hline Phyenylalanine & $\mathrm{BDL}^{*}$ & $\mathrm{BDL}^{*}$ & $\mathrm{BDL}^{*}$ & $\mathrm{BDL}^{*}$ \\
\hline Isoleucine & BDL* & 23.60 & BDL* & 22.60 \\
\hline Leucine & 0.40 & 1.20 & BDL* & 0.50 \\
\hline Lysine & BDL* & 0.30 & BDL* & BDL* \\
\hline Proline & 88.20 & BDL* & 59.30 & 19.50 \\
\hline
\end{tabular}

* Below Detection Limit 
Table.2 UHPLC Validation Data for Amino Acid Standards

\begin{tabular}{|c|r|r|r|r|l|r|}
\hline Peak\# & $\begin{array}{l}\text { Ret. } \\
\text { Time }\end{array}$ & \multicolumn{1}{l|}{ Area } & Height & Area \% & Name & $\begin{array}{l}\text { Conc. } \\
(\mathbf{m g} / \mathbf{L})\end{array}$ \\
\hline 1. & 0.453 & 16377 & 1670 & 0.253 & L-Aspartic acid & 2.5 \\
\hline 2. & 0.667 & 129126 & 31880 & 1.992 & L-Glutamic acid & 2.5 \\
\hline 3. & 0.97 & 238650 & 53580 & 3.682 & L-Serine & 2.5 \\
\hline 4. & 1.418 & 16918 & 3609 & 0.261 & L-Histidine & 2.5 \\
\hline 5. & 1.915 & 195540 & 38754 & 3.017 & Glycine & 2.5 \\
\hline 6. & 2.372 & 12009 & 1332 & 0.185 & Threonine & 2.5 \\
\hline 7. & 2.772 & 14353 & 1664 & 0.221 & Arginine & 2.5 \\
\hline 8. & 3.16 & 3286630 & 316310 & 50.702 & Alanine & 2.5 \\
\hline 9. & 3.693 & 429450 & 31822 & 6.276 & Tyrosine & 2.5 \\
\hline 10. & 5.185 & 444133 & 36688 & 6.852 & Cystine & 1.25 \\
\hline 11. & 6.269 & 144847 & 11371 & 2.235 & Valine & 2.5 \\
\hline 12. & 7.847 & 39286 & 3306 & 0.606 & Methionine & 2.5 \\
\hline 13. & 8.579 & 271763 & 49250 & 4.192 & Phenylalanine & 2.5 \\
\hline 14. & 8.986 & 795899 & 124754 & 12.278 & Isoleucine & 2.5 \\
\hline 15. & 9.848 & 125000 & 26467 & 1.928 & Leucine & 2.5 \\
\hline 16. & 9.988 & 105799 & 25163 & 1.632 & Lysine & 2.5 \\
\hline 17. & 11.198 & 239071 & 32888 & 3.688 & Proline & 2.5 \\
\hline
\end{tabular}

Table.3 UHPLC Validation Data for Amino Acid Content in Monascus purpureus (MTCC 369)

\begin{tabular}{|c|c|c|c|c|c|c|}
\hline Peak\# & Ret. Time & Area & Height & Area\% & Name & $\begin{array}{l}\text { Conc. } \\
\text { (mg/L) }\end{array}$ \\
\hline 1. & 0.433 & 14355 & 4742 & 1.041 & Aspartic Acid & 5.90 \\
\hline 2. & 0.703 & 6505 & 1171 & 0.472 & Glutamic Acid & 1.30 \\
\hline 3. & 1.364 & 11190 & 2174 & 0.812 & Serine & 1.80 \\
\hline 4. & 2.414 & 30597 & 3592 & 2.22 & Histldine & 0.60 \\
\hline 5. & No peak is Detected. & 0 & 0 & 0 & Glyeine & BDL \\
\hline 6. & No peak is Detected. & 0 & 0 & 0 & L-Threonine & BDL \\
\hline 7. & No peak is Detected. & 0 & 0 & 0 & Arginine & BDL \\
\hline 8. & No peak is Detected. & 0 & 0 & 0 & Alanine & BDL \\
\hline 9. & 5.653 & 43681 & 4135 & 3.169 & Tyrosine & 5.70 \\
\hline 10. & 6.329 & 6703 & 1008 & 0.486 & Cystine & 0.80 \\
\hline 11. & 6.648 & 27494 & 3309 & 1.995 & Valline & 2.40 \\
\hline 12. & 7.104 & 4622 & 120 & 0.335 & Methionine & BDL \\
\hline 13. & No peak is Detected. & 0 & 0 & 0 & Phyenylalanine & BDL \\
\hline 14. & No peak is Detected. & 0 & 0 & 0 & Isoleucine & BDL \\
\hline 15. & 8.735 & 2223 & 392 & 0.161 & Leucine & 0.40 \\
\hline 16. & No peak is Detected. & 0 & 0 & 0 & Lysine & BDL \\
\hline 17. & 10.944 & 1232895 & 95424 & 89.439 & Proline & 88.20 \\
\hline
\end{tabular}

*BDL- Below Detection Limit 
Table.4 UHPLC Validation Data for Amino Acid Content in Monascus purpureus (MTCC 410)

\begin{tabular}{|r|c|c|c|c|l|c|}
\hline \multicolumn{1}{|l|}{ Peak\# } & Ret. Time & Area & Height & Area\% & Name & Conc. $(\mathbf{m g} / \mathbf{L})$ \\
\hline 1 & No peak is detected. & 0 & 0 & 0 & Aspartic Acid & BDL \\
\hline 2 & 0.695 & 6851 & 1340 & 3.018 & Glutamic Acid & 1.40 \\
\hline 3 & 1.338 & 8734 & 2047 & 3.848 & Serine & 1.40 \\
\hline 4 & 2.371 & 33144 & 3765 & 14.601 & Histldine & 0.60 \\
\hline 5 & 3.278 & 1755 & 140 & 0.773 & Glyeine & 2.40 \\
\hline 6 & No peak is detected. & 0 & 0 & 0 & L-Threonine & BDL \\
\hline 7 & 4.542 & 2585 & 316 & 1.139 & Arginine & 0.30 \\
\hline 8 & No peak is detected. & 0 & 0 & 0 & Alanine & BDL \\
\hline 9 & 5.633 & 38163 & 4728 & 16.812 & Tyrosine & 5.00 \\
\hline 10 & 6.28 & 2896 & 474 & 1.276 & Cystine & 0.30 \\
\hline 11 & 6.658 & 22158 & 2049 & 9.761 & Valline & 2.00 \\
\hline 12 & 7.104 & -2101 & 107 & -0.925 & Methionine & BDL \\
\hline 13 & No peak is detected. & 0 & 0 & 0 & Phyenylalanine & BDL \\
\hline 14 & 7.912 & 94857 & 3115 & 41.788 & Isoleucine & 23.60 \\
\hline 15 & 8.525 & 6019 & 692 & 2.652 & Leucine & 1.20 \\
\hline 16 & 8.763 & 2275 & 384 & 1.002 & Lysine & 0.30 \\
\hline 17 & No peak is detected. & 0 & 0 & 0 & Proline & BDL \\
\hline
\end{tabular}

*BDL- Below Detection Limit

Table.5 UHPLC Validation Data for Amino Acid Content in Monascus ruber (MTCC 1880)

\begin{tabular}{|r|c|r|r|r|l|c|}
\hline Peak\# & Ret. Time & Area & Height & Area\% & Name & $\begin{array}{c}\text { Conc. } \\
\text { (mg/L) }\end{array}$ \\
\hline 1 & 0.433 & 15787 & 5430 & 1.538 & Aspartic Acid & 6.50 \\
\hline 2 & 0.703 & 10433 & 2131 & 1.016 & Glutamic Acid & 2.20 \\
\hline 3 & 1.354 & 8475 & 1391 & 0.826 & Serine & 1.40 \\
\hline 4 & 2.371 & 48047 & 3305 & 4.68 & Histldine & 0.90 \\
\hline 5 & 3.165 & 2044 & 80 & 0.199 & Glyeine & 2.80 \\
\hline 6 & No peak is detected. & 0 & 0 & 0 & L-Threonine & BDL \\
\hline 7 & No peak is detected. & 0 & 0 & 0 & Arginine & BDL \\
\hline 8 & No peak is detected. & 0 & 0 & 0 & Alanine & BDL \\
\hline 9 & 5.628 & 26160 & 2428 & 2.548 & Tyrosine & 3.40 \\
\hline 10 & 6.262 & 1488 & 287 & 0.145 & Cystine & 0.20 \\
\hline 11 & 6.654 & 27709 & 2069 & 2.699 & Valline & 2.50 \\
\hline 12 & 7.052 & 54345 & 3187 & 5.294 & Methionine & 9.70 \\
\hline 13 & No peak is detected. & 0 & 0 & 0 & Phyenylalanine & BDL \\
\hline 14 & No peak is detected. & 0 & 0 & 0 & Isoleucine & BDL \\
\hline 15 & No peak is detected. & 0 & 0 & 0 & Leucine & BDL \\
\hline 16 & No peak is detected. & 0 & 0 & 0 & Lysine & BDL \\
\hline 17 & 11.024 & 828383 & 66195 & 80.693 & Proline & 59.30 \\
\hline
\end{tabular}

*BDL- Below Detection Limit 
Table.6 UHPLC Validation Data for Amino Acid Content in Monascus ruber (MTCC 2326)

\begin{tabular}{|r|c|r|r|r|c|c|}
\hline Peak\# & Ret. Time & Area & Height & Area\% & Name & $\begin{array}{l}\text { Conc. } \\
\text { (mg/L) }\end{array}$ \\
\hline 1 & No peak is detected. & 0 & 0 & 0 & Aspartic Acid & BDL \\
\hline 2 & 0.686 & 9000 & 1735 & 1.396 & Glutamic Acid & 1.90 \\
\hline 3 & 1.311 & 10754 & 1979 & 1.668 & Serine & 1.70 \\
\hline 4 & 2.315 & 53550 & 3355 & 8.306 & Histldine & 1.00 \\
\hline 5 & 3.095 & 1835 & 138 & 0.285 & Glyeine & 2.50 \\
\hline 6 & No peak is detected. & 0 & 0 & 0 & L-Threonine & BDL \\
\hline 7 & No peak is detected. & 0 & 0 & 0 & Arginine & BDL \\
\hline 8 & No peak is detected. & 0 & 0 & 0 & Alanine & BDL \\
\hline 9 & 5.605 & 30813 & 3069 & 4.779 & Tyrosine & 4.00 \\
\hline 10 & 6.258 & 2954 & 324 & 0.458 & Cystine & 0.30 \\
\hline 11 & 6.654 & 48841 & 2929 & 7.576 & Valline & 4.30 \\
\hline 12 & 7.019 & 116579 & 6159 & 18.083 & Methionine & 20.80 \\
\hline 13 & No peak is detected. & 0 & 0 & 0 & Phyenylalanine & 0.00 \\
\hline 14 & 7.946 & 90891 & 3078 & 14.098 & Isoleucine & 22.60 \\
\hline 15 & 8.705 & 2597 & 322 & 0.403 & Leucine & 0.50 \\
\hline 16 & No peak is detected. & 0 & 0 & 0 & Lysine & BDL \\
\hline 17 & 11.056 & 272379 & 33757 & 42.248 & Proline & 19.50 \\
\hline
\end{tabular}

*BDL- Below Detection Limit

Figure.1 UHPLC Chromatogram of Amino Acid Standards

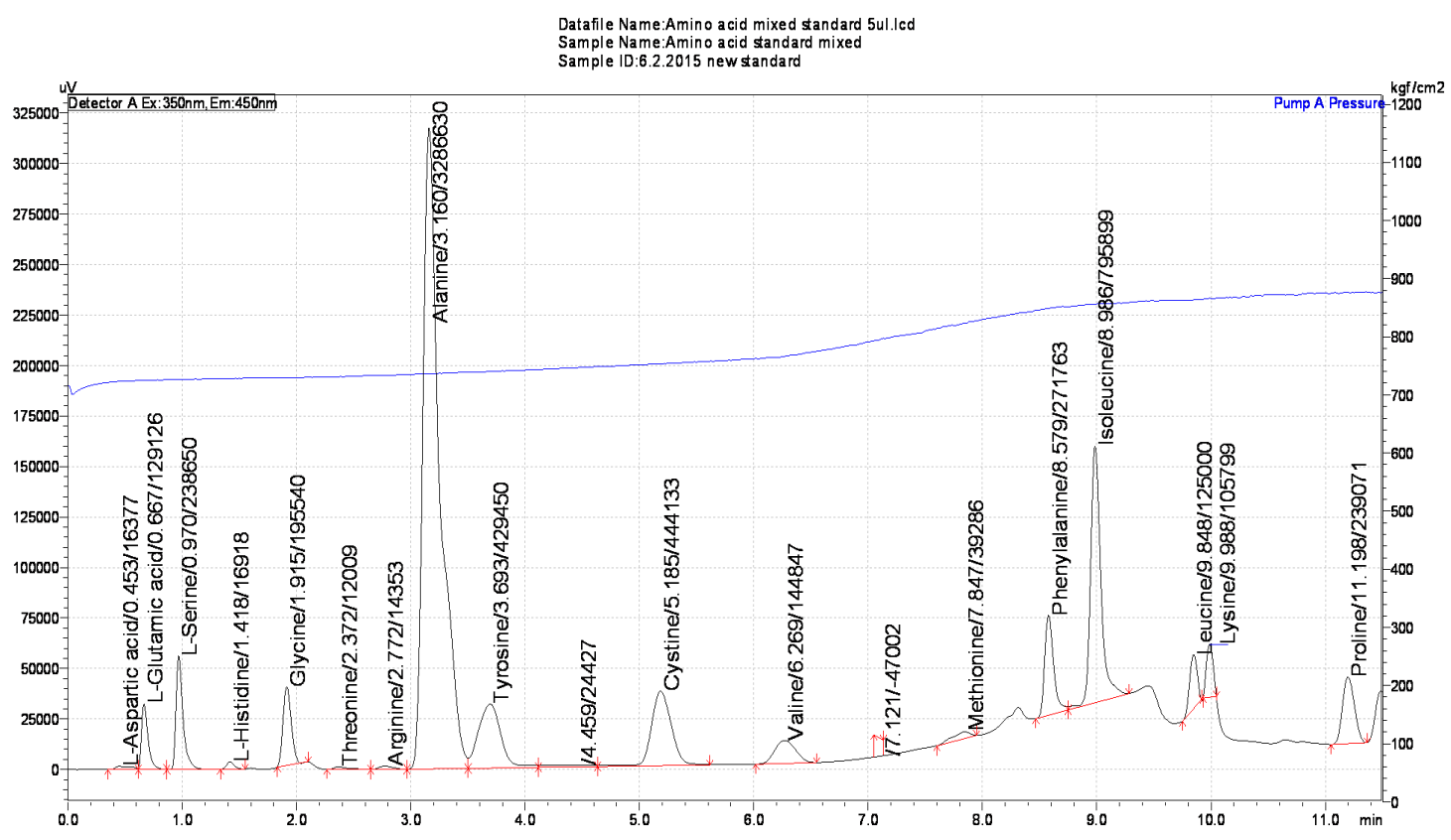


Figure.2 UHPLC Chromatogram of Comparative Amino acid Standards

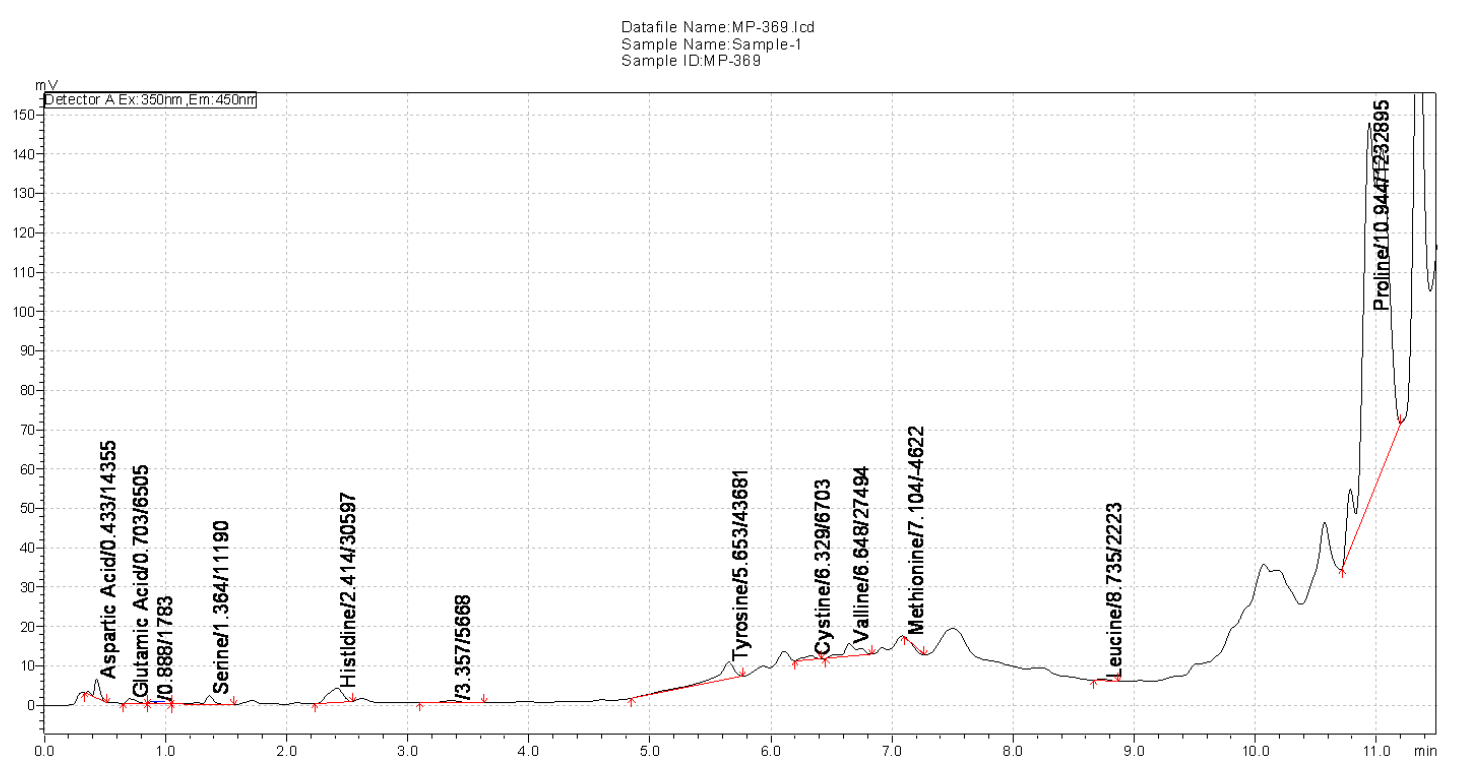

Figure.3 UHPLC Chromatogram of Amino Acid Content in Monascus purpureus (MTCC 410)

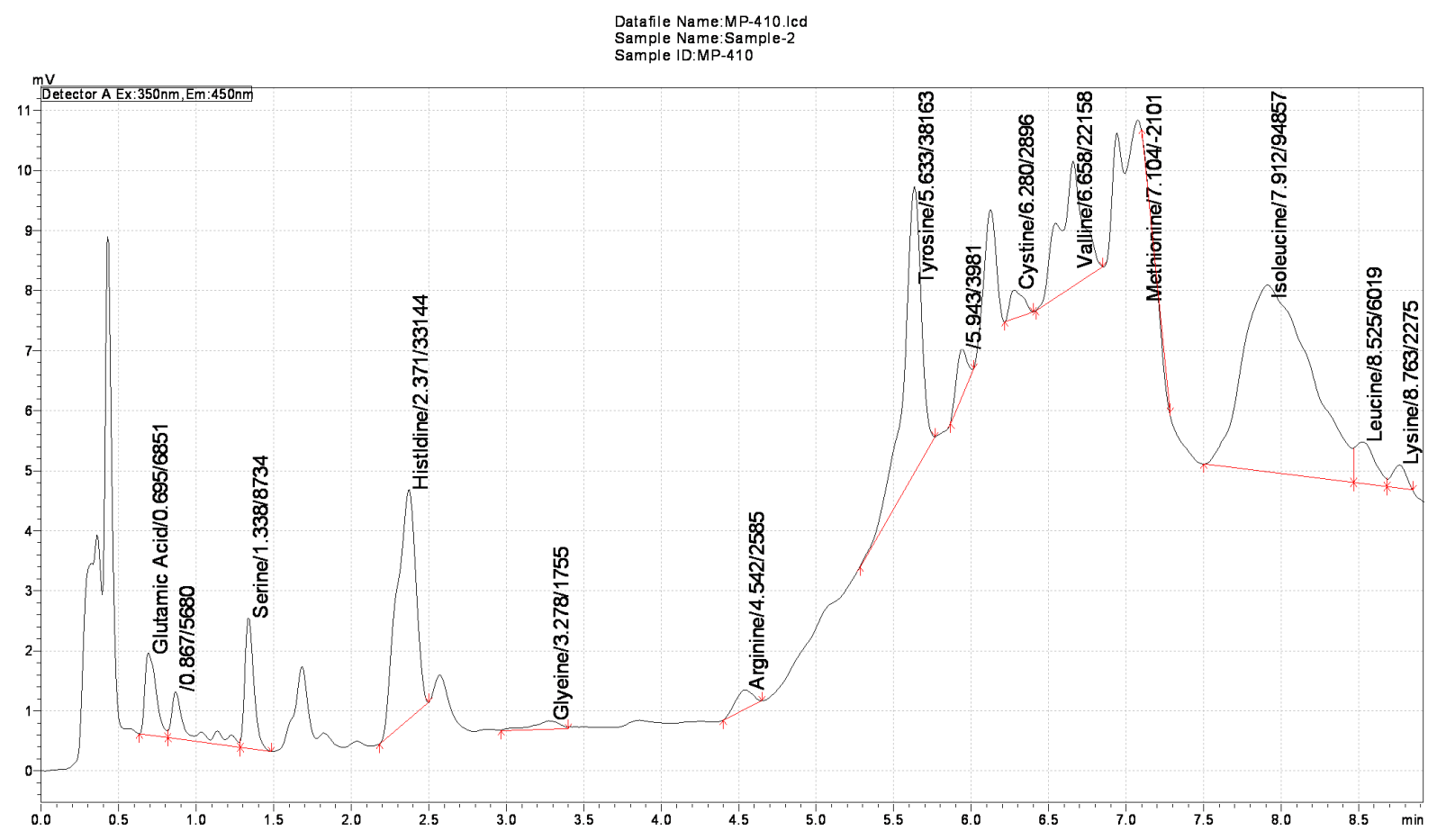


Figure.4 UHPLC Chromatogram of Amino acid Content in Monascus ruber (MTCC 1880)

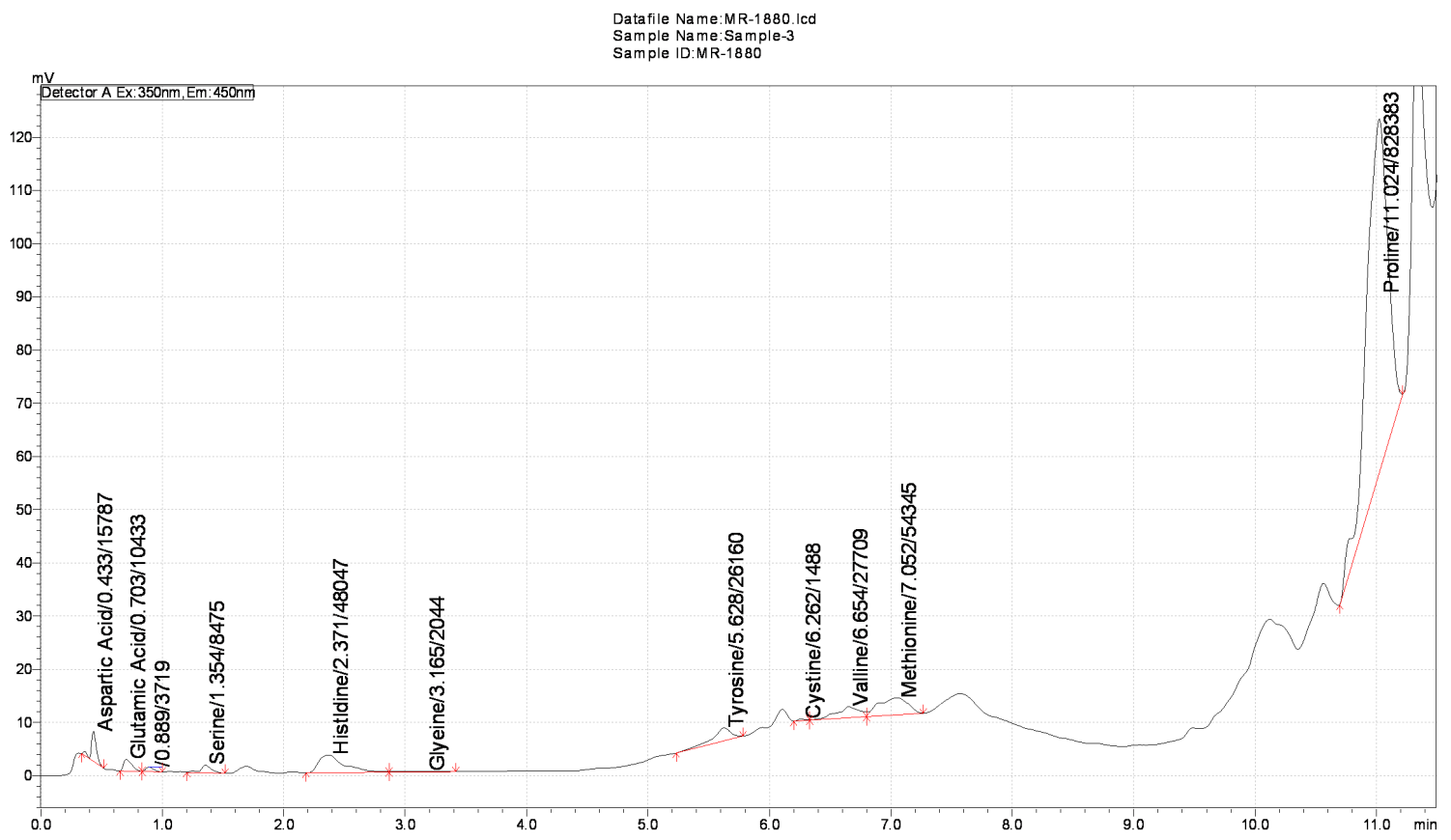

Figure.5 UHPLC Chromatogram of Amino Acid Content in Monascus ruber (MTCC 2326)

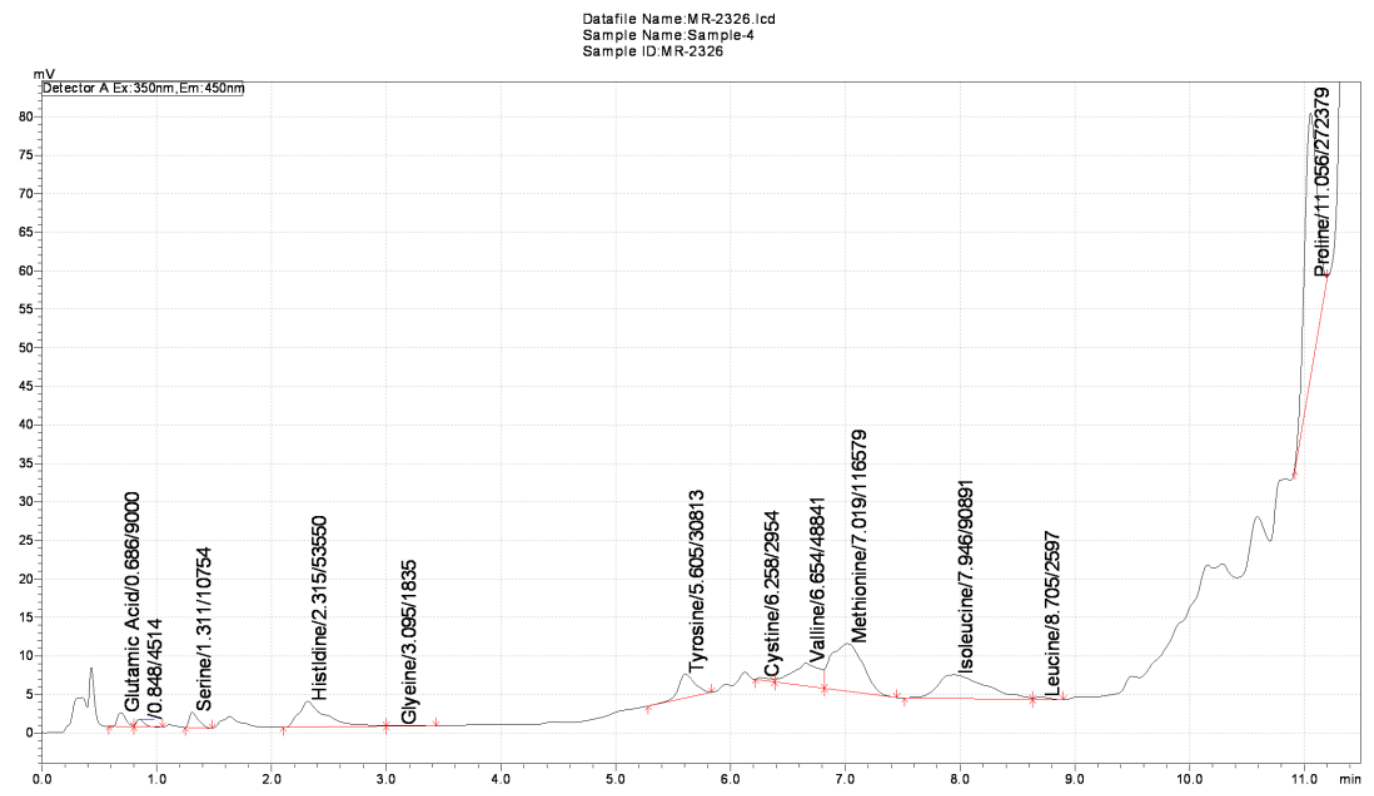


In conclusion, the attainment of this study is a reliable and high throughput method for the separation and quantification of amino acids in the monascus pigments from Monascus purpureus (MTCC 410), Monascus purpureus (MTCC 369), Monascus ruber (MTCC 1880) and Monascus ruber (MTCC 2326). The determination of amino acids using UHPLC in combination with OPA as a derivatization agent provides a very useful method.

\section{Acknowledgement}

The authors would like to thank Dr.M.Loganathan, Director i/C, Indian Institute of Crop Processing Technology, Ministry of Food Processing Industries, Govt. of India, Thanjavur-613 005 TamilNadu, India for their support of this study.

\section{References}

Armağan Önal, Serife Evrim Kepekci Tekkeli, Cem Önal. 2013. A review of the liquid chromatographic methods for the determination of biogenic amines in foods. Food Chemistry, 138(1): 509515.

Bomke, S., Seiwert, B., Dudek, L., Effkemann, S., Karst, U. 2009. Determination of biogenic amines in food samples using derivatization followed by liquid chromatography/ mass spectrometry. Analytical and Bioanalytical Chemistry, 393(1): 247256.

Onal, A. 2007. A review: current analytical methods for the determination of biogenic amines in foods. Food Chemistry, 103(4): 1475-1486.

Perucho, R., Gonzalo-Gobernado, E., Bazan, M.J., Casarejos, A., Jiménez crig, M.J., Asensio, A.S., Herranz. 2015. Optimal excitation and emission wavelengths to analyze amino acids and optimize neurotransmitters quantification using precolumn OPA-derivatization by HPLC. Amino Acids, doi10.1007/s00726-015-1925-1.

Rouba Horanni, Ulrich, H., Engelhardt. 2013. Determination of amino acids in white, green, black, oolong, pu-erh teas and tea products. J. Food Composition and Analysis, 31(1): 94-100.

Shimadzu Corporation. 2012. Application News No.L432, High Speed, High Resolution Analysis (Part 44) Analysis of Pre-Column Derivatized Amino Acids by SIL-30AC Autosampler. First Edition.

Zhaolai Dai, Zhenlong Wu, Sichao Jia, Guoyao Wu. 2014. Analysis of amino acid composition in proteins of animal tissues and foods as precolumn $o$-phthaldialdehyde derivatives by HPLC with fluorescence detection. J. Chromatography B, 964: 116-127.

\section{How to cite this article:}

Vidyalakshmi, R., Paranthaman, R. and Sureshkumar, K. 2016. Simultaneous Determination of 17 Amino Acids in Microbial Pigments from Monascus spp By UHPLC Amino Acid Analyser Using Pre-Column Derivatization. Int.J.Curr.Microbiol.App.Sci. 5(3): 354-362. doi: http://dx.doi.org/10.20546/ijcmas.2016.503.042 